\title{
Experimental and theoretical investigation of phosphorus in-situ doping of germanium epitaxial layers
}

\author{
Hyun-Yong Yu ${ }^{\mathrm{a}, *}$, Enes Battal ${ }^{\mathrm{b}}$, Ali Kemal Okyay ${ }^{\mathrm{b}}$, Jaewoo Shim ${ }^{\mathrm{c}}$, Jin-Hong Park ${ }^{\mathrm{c}, * *}$, \\ Jung Woo Baek ${ }^{\mathrm{d}}$, Krishna C. Saraswat ${ }^{\mathrm{e}}$ \\ ${ }^{a}$ School of Electrical Engineering, Korea University, Seoul 136-713, Republic of Korea \\ ${ }^{\mathrm{b}}$ Department of Electrical and Electronic Engineering, Bilkent University, 06800 Ankara, Turkey \\ ${ }^{c}$ School of Electronics and Electrical Engineering, Sungkyunkwan University, Suwon 440-746, Republic of Korea \\ ${ }^{\mathrm{d}}$ Department of Systems Management Engineering, Sungkyunkwan University, Suwon 440-746, Republic of Korea \\ e Department of Electrical Engineering, Stanford University, Stanford, CA 94305, United States
}

\section{A R T I C L E I N F O}

\section{Article history:}

Received 26 November 2012

Received in revised form

6 February 2013

Accepted 26 February 2013

Available online 14 March 2013

Keywords:

In-situ

Germanium

Diffusivity

Activation energy

Phosphorus

\begin{abstract}
A B S T R A C T
We investigate phosphorus in-situ doping characteristics in germanium (Ge) during epitaxial growth by spreading resistance profiling analysis. In addition, we present an accurate model for the kinetics of the diffusion in the in-situ process, modeling combined growth and diffusion events. The activation energy and pre-exponential factor for phosphorus (P) diffusion are determined to be $1.91 \mathrm{eV}$ and $3.75 \times 10^{-5} \mathrm{~cm}^{2} / \mathrm{s}$. These results show that $\mathrm{P}$ in-situ doping diffusivity is low enough to form shallow junctions for high performance Ge devices.
\end{abstract}

(c) 2013 Elsevier B.V. All rights reserved.

\section{Introduction}

Compared to silicon ( $\mathrm{Si}$ ), higher carrier mobility and carrier injection velocity of germanium $(\mathrm{Ge})$ makes it an attractive candidate for electronic devices. In addition, Ge offers a wider absorption window, specially, toward the near infrared region, where Si shows weak or no optical absorption at all. The formation of p-type shallow junctions in Ge (films/layers/substrates) has been successfully demonstrated via ion-implantation technique and in-situ doping during epitaxial growth for boron (B) [1,2] with concentrations above $2 \times 10^{18} \mathrm{~cm}^{-3}$, the solid solubility of B in Ge. However, in the case of n-type Ge junction formation, significant effort is still underway to realize high concentrations and shallow junctions. Arsenic (As) is known to have low solid solubility and high

\footnotetext{
* Corresponding author. Present address: School of Electrical Engineering, Korea University, Anam-dong, Seongbuk-ku, Seoul 136-713, Republic of Korea. Tel.: +822 32904830.

** Corresponding author. Present address: School of Electronic and Electrical Engineering, Sungkyunkwan University, Cheoncheon-dong, Jangan-gu, Suwon-si, Gyeonggi-do 440-746, Republic of Korea. Tel.: +82 312994951.

E-mail addresses: yuhykr@korea.ac.kr (H.-Y. Yu), jhpark9@skku.edu (J.-H. Park).
}

activation energy ( $2.71 \mathrm{eV}$ ) [3] because its large radius makes it difficult to substitute for Ge atoms [4,5]. In addition, since its high diffusion coefficient $\left(32 \mathrm{~cm}^{2} / \mathrm{s}\right)$ [4] hinders the formation of $\mathrm{n}^{+}$ shallow junctions in Ge, phosphorus $(\mathrm{P})$, which has relatively high solubility, low activation energy, and low diffusivity, has been widely used. Ion implantation of P can be used to achieve chemical concentrations [6] high $10^{19} \mathrm{~cm}^{-3}$ regime, but implant damages $[7,8]$ induce transient enhanced diffusion (TED) hindering formation of shallow junctions in addition to dose losses [6-9], caused by out-diffusion. Doping by conventional furnace diffusion of P leads to deep junctions due to long in-diffusion [5]. In contrast to these doping approaches, in-situ doping technique avoids any implantation-related damages and deep in-diffusions, and consequently provides fairly shallow junctions while offering high electrically activated concentrations [10,11] at low processing temperatures. We recently demonstrated an abrupt and boxshaped high quality $\mathrm{Ge} \mathrm{n}^{+} / \mathrm{p}$ junction diode with in-situ doping technique the result of which is reported elsewhere [12]. In order to estimate accurately junction depth and shape when forming n-type shallow junctions in Ge, accurate dopant diffusion modeling is required. Although there are a few theoretical studies about $\mathrm{P}$ diffusion in $\mathrm{Ge}[14,15]$, by simulations, such analyses are performed 
under the assumption that $\mathrm{P}$ atoms are doped by ion implantation method and a simulation model related to $\mathrm{P}$ in-situ doping technique is not well established yet. Therefore, in this letter, we investigate the electrical characteristics of $P$ in-situ doped epitaxial Ge layers and follow with a discussion of theoretical diffusion model that can accurately predict our experimental findings.

\section{Experiments}

After the standard Si wafer cleaning process, a p-type (100) Si wafer was immediately loaded into a cold wall Applied Materials Centura RP-CVD epitaxial reactor. A hydrogen bake at $900{ }^{\circ} \mathrm{C}$ was carried out to make sure that no native oxide remained on the $\mathrm{Si}$ surface because native oxide on Si wafer prevents Ge film growth on Si substrate. A very thin Si epi layer was first grown using dichlorosilane (DCS) at $700{ }^{\circ} \mathrm{C}$ to improve the final film quality. This initial growth was followed by annealing in $\mathrm{H}_{2}$ ambient for $30 \mathrm{~min}$ at $825^{\circ} \mathrm{C}$. Next two Ge buffer growth steps were performed at $400{ }^{\circ} \mathrm{C}$ with the growth rate of $\sim 30 \mathrm{~nm} / \mathrm{min}$, also followed by anneal in $\mathrm{H}_{2}$ ambient at $825^{\circ} \mathrm{C}$. The hydrogen annealing process reduced Ge surface roughness by re-flowing the Ge atoms [16]. Then, we performed the final growth step for intrinsic Ge layer at $600{ }^{\circ} \mathrm{C}$ with the growth rate of $\sim 60 \mathrm{~nm} / \mathrm{min}$. This intrinsic Ge epitaxial layer showed p-type $1 \times 10^{14} \mathrm{~cm}^{-3}$ of electrically activated concentration. Before growing the doped layer, the dopant gas line is purged with diluted $1 \%$ phosphine to avoid the dopant crosscontamination at the stabilization step. The final Ge layer was grown at $400{ }^{\circ} \mathrm{C}-600{ }^{\circ} \mathrm{C}$ at $8 \mathrm{~Pa}$ on the intrinsic Ge layer, in-situ doped with diluted $1 \%$ phosphine for 2 min to form a $\mathrm{n}^{+}$Ge layer.

\section{Results and discussion}

On an intrinsic Ge virtual substrate, in-situ doping of $\mathrm{P}$ is performed by $\mathrm{PH}_{3}$ and $\mathrm{GeH}_{4}$ flow for 2 min at the maximum activated doping condition. The mass flow ratios of $\mathrm{F}\left(\mathrm{PH}_{3}\right) / \mathrm{F}\left(\mathrm{GeH}_{4}\right)$ are 0.007 , 0.009 , and 0.01 at the growth temperatures of $400{ }^{\circ} \mathrm{C}, 500{ }^{\circ} \mathrm{C}$, and $600{ }^{\circ} \mathrm{C}$ respectively. Spreading resistance profiling (SRP) measurements on grown samples are obtained. Extracted concentration of $P$ atoms versus depth from the surface is plotted in Fig. 1 at the

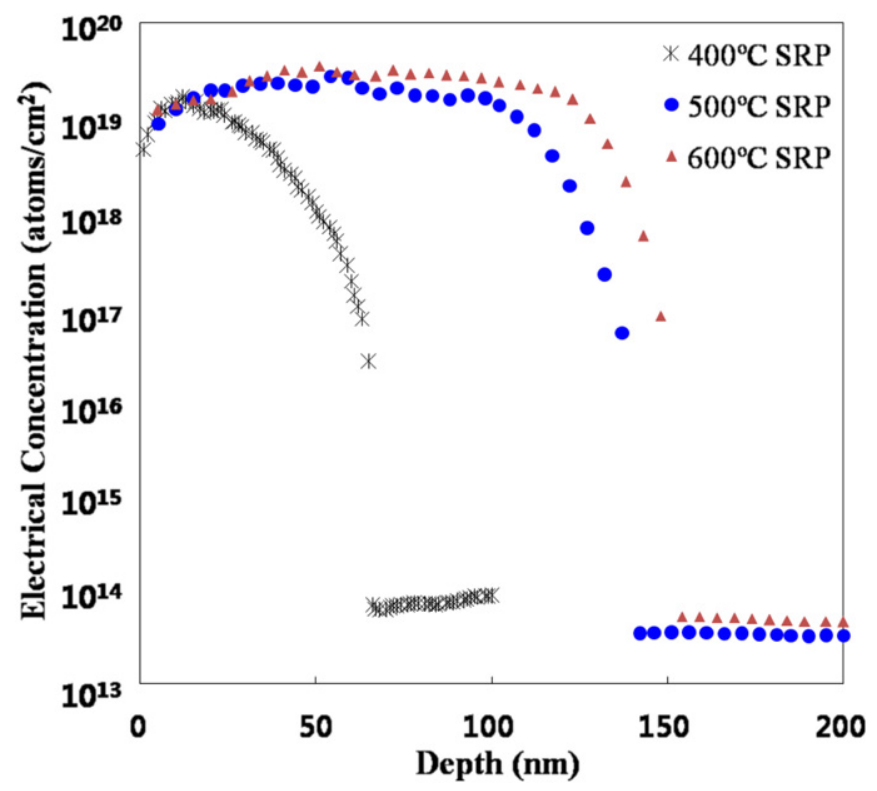

Fig. 1. SRP depth profiles of $\mathrm{P}$ in $400{ }^{\circ} \mathrm{C}, 500{ }^{\circ} \mathrm{C}$, and $600{ }^{\circ} \mathrm{C}$ in-situ doped Ge layer growth for 2 min. growth temperatures of $400{ }^{\circ} \mathrm{C}-600{ }^{\circ} \mathrm{C}$. Deeper diffusion of $\mathrm{P}$ atoms was observed as the deposition temperature increases. The profile exhibits an abrupt and box-shaped junction formation with a slope for the decay of $P$ concentration, $13.7 \mathrm{~nm} /$ decade. In addition, the electrically activated dopant profile near the surface have positive slopes such that the electrically activated dopant profiles near the surface is not at maximum because of the shorter anneal time of the deposited phosphorus atoms in this region compared to inner region.

The existing models in literatures $[3,6,7,14,15]$ predicting the diffusion kinetics of $P$ in Ge use error-function profiles. In such models, diffusion is from either an initial dopant profile by ion implantation or assuming a constant dose source at the surface. However, the actual epitaxial growth and in-situ doping process is modeled as consecutive discrete events. Fig. 2 shows the proposed growth model for in-situ doping process. During the in-situ doping process, dopants are provided at the growing surface, and at the same time, diffuse into the substrate. In order to take the effect of a moving surface frontier into account, the growth process is modeled by discrete consecutive cycles. Each cycle is composed of growth phase and diffusion phase. During the growth phase, highly doped thin Ge layer of thickness $\Delta x$ is assumed to be deposited on the surface. Dopants are diffused during the diffusion phase, and the new doping profile after a short time interval $\Delta t$ is obtained. A single cycle models in-situ doping growth and dopant diffusion during the short time interval $\Delta t$, so growth thickness $\Delta x$ should be $v \cdot \Delta t$, where $v$ is the growth rate of Ge layer $(\mathrm{nm} / \mathrm{sec})$. To model the dopant diffusion during the time interval $\Delta t$, first, we divide the Ge substrate into very thin layers. If the thickness of the each layer is thin enough, then the doping profile in each layer can be considered as a delta function.

Fick's Second Law is applied to numerically calculate the resulting diffuse profile. The solution of a cycle is used as the initial condition for the subsequent cycle in time.

$\frac{\partial C}{\partial t}=\frac{\partial}{\partial x}\left(D \frac{\partial C}{\partial x}\right)$

Due to high doping concentrations required in device applications, concentration-dependent-diffusion and electric-field (Efield) effects were included in our model. The following relations were used for second order concentration dependency and E-field effects:

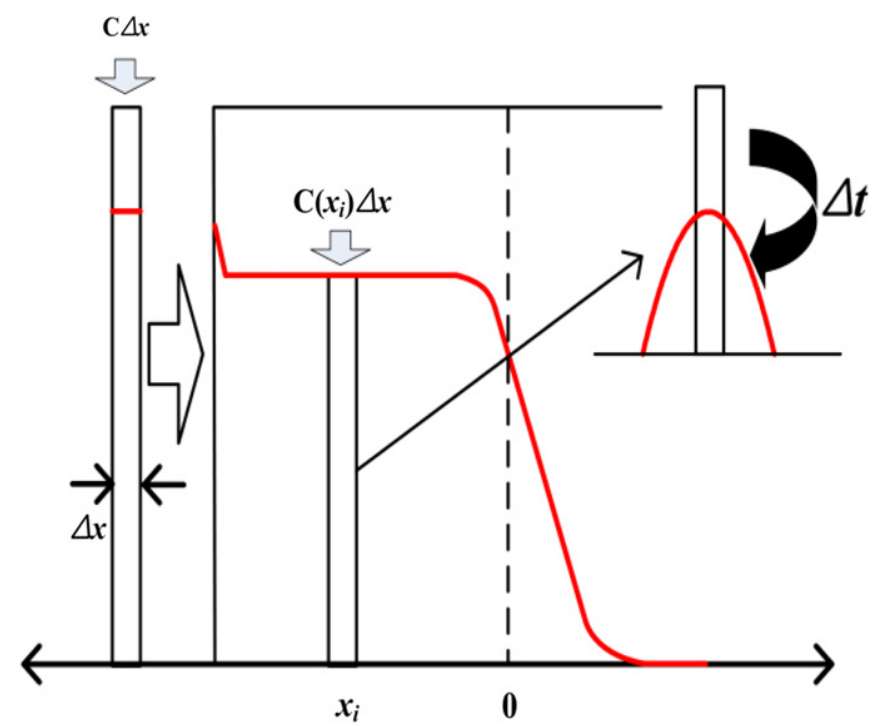

Fig. 2. Schematic of in-situ growth model. 


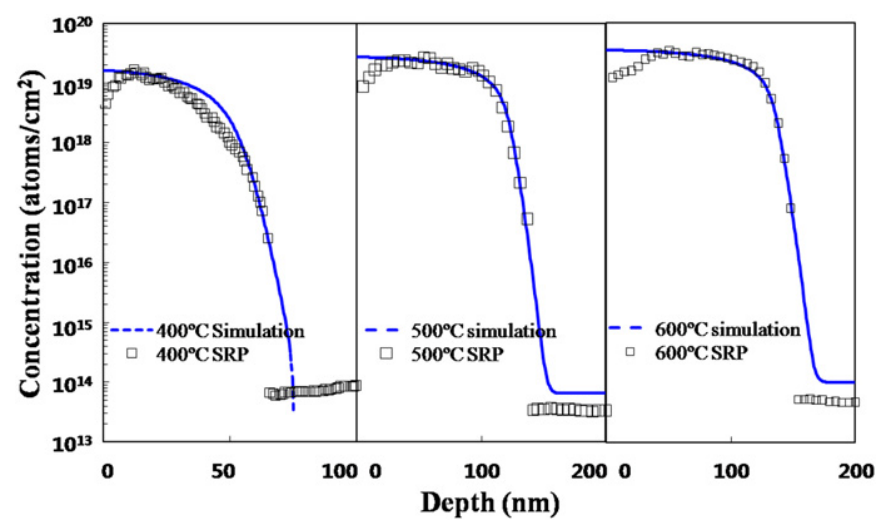

Fig. 3. Phosphorus $(\mathrm{P})$ concentration profiles of experimental and simulated data at $400{ }^{\circ} \mathrm{C}, 500{ }^{\circ} \mathrm{C}$ and $600{ }^{\circ} \mathrm{C}$.

$D=D^{0}+D^{-}\left(\frac{n}{n_{i}}\right)+D^{--}\left(\frac{n}{n_{i}}\right)^{2}$

$\frac{\partial C}{\partial t}=\frac{\partial}{\partial x}\left(D\left[\frac{\partial C}{\partial}\right]\right)+\frac{\partial}{\partial x}\left(D C \frac{\partial}{\partial x}\left[\ln \left(\frac{n}{n_{i}}\right)\right]\right)$

where the first term $\partial / \partial x(D[\partial C / \partial])$ is related to basic Fick's Second Law and the second term $\partial / \partial x\left(D C \partial / \partial x\left[\ln \left(n / n_{i}\right)\right]\right)$ is related to the Efield effect. In order to solve these equations, the derived solution in Asaithambi [13] and finite-difference method is used.

The concentrations of species obtained by SRP data are electrically active concentrations. It was shown that the electrical activation levels for $400^{\circ} \mathrm{C}, 500^{\circ} \mathrm{C}$, and $600^{\circ} \mathrm{C}$ in-situ P-doped Ge epitaxy layers were $30 \%, 65 \%$, and $100 \%$ respectively [12]. In the model described, the chemical concentration of $\mathrm{P}$ in $\mathrm{Ge}$ was used by scaling the measured electrically active concentration by the level of activation at each growth temperature. As shown in Fig. 3, the resulting profiles calculated numerically with our model and the experimental results are in very good agreement at $400^{\circ} \mathrm{C}, 500^{\circ} \mathrm{C}$, and $600^{\circ} \mathrm{C}$ respectively.

At a given growth temperature, the doubly charged diffusivity, $\mathrm{D}^{--}$, dominates due to the quadratic dependence on the carrier concentration. This supports the presence of concentration

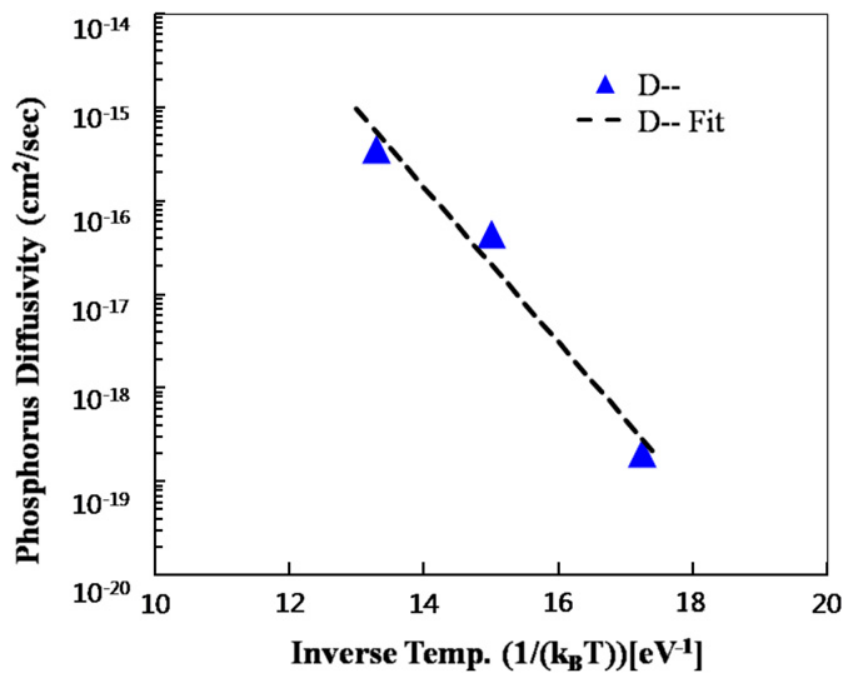

Fig. 4. Plot of diffusivity coefficients $\left(\mathrm{D}^{-}{ }^{-}\right.$) at different temperatures. For $\mathrm{D}^{-}{ }^{-}$, activation energy is $1.91 \mathrm{eV}$, and diffusivity pre-factor is $3.75 \times 10^{-5} \mathrm{~cm}^{2} / \mathrm{s}$. dependent effects in addition to a sharp decaying tail of the measured profile. The junction depth, therefore, is governed by the doubly charged diffusivity of $\mathrm{P}$ atoms. Fig. 4 plots the extracted doubly charged diffusivity coefficient and corresponding Arrhenius curve to fit experimental data. It is shown that the activation energy and pre-exponential factor for phosphorus diffusion are determined to $1.91 \mathrm{eV}$ and $3.75 \times 10^{-5} \mathrm{~cm}^{2} / \mathrm{s}$. The activation energy corresponding to the doubly charged diffusivity, $\mathrm{D}^{-}{ }^{-}$, in this work is slightly lower than that observed in Chui et al. (2.07 eV) [5], Carroll et al. (2.3 eV) [14] and Tsouroutas et al. $(2.69 \mathrm{eV})$ [15]. Chui et al. and Carroll et al. investigated the concentration dependent diffusivity of $\mathrm{P}$ in Ge when the dopants are introduced by ion-implantation technique. The pre-factor of the second order diffusivity coefficient obtained in this work is dramatically lower than that reported by Chui et al. $\left(4.38 \times 10^{-2} \mathrm{~cm}^{2} / \mathrm{s}\right)$ and Carroll et al. $\left(1.85 \times 10^{-2} \mathrm{~cm}^{2} / \mathrm{s}\right)$. Therefore, in-situ doping technique can offer the low diffusivity unattainable by earlier work [5,14], paving the way for fabricating shallow n-type junctions and devices with high levels of electrically active $P$ in Ge.

\section{Conclusion}

We investigated characteristics of $\mathrm{P}$ in-situ doped Ge obtained by epitaxial growth. The accurate modeling of $\mathrm{P}$ in-situ doping process was achieved with the activation energy of $1.91 \mathrm{eV}$ and preexponential factor of $3.75 \times 10^{-5} \mathrm{~cm}^{2} / \mathrm{s}$. Phosphorus in-situ doping diffusivity is low enough to form shallow junction devices with high performance in Ge substrate.

\section{Acknowledgment}

This work was performed at the Stanford Nanofabrication Facility (SNF) and was supported by MARCO Interconnect Focus Centers, the Stanford University INMP program, and the Korea University research fund. This work was supported by Basic Science Research Program through the National Research Foundation of Korea (NRF) funded by the Ministry of Education, Science, and Technology (NRF-2011-0007997).

\section{References}

[1] S. Uppal, A.F.W. Willoughby, J.M. Bonar, N.E.B. Cowern, T. Grasby, R.J.H. Morris, M.G. Dowsett, Diffusion of boron in germanium at $800-900^{\circ} \mathrm{C}$, J. Appl. Phys. 96 (2004) 1376

[2] S. Uppal, A.F.W. Willoughby, J.M. Bonar, A.G.R. Evans, Nick E.B. Cowern, R. Morris, M.G. Dowsett, Diffusion of ion-implanted boron in germanium, J. Appl. Phys. 90 (2001) 4293.

[3] S. Brotzmann, H. Bracht, Intrinsic and extrinsic diffusion of phosphorus, arsenic, and antimony in germanium, J. Appl. Phys. 103 (2008) 033508.

[4] M. Koike, Y. Kamata, T. Ino, D. Hagishima, K. Tatsumura, M. Koyama, A. Nishiyama, Diffusion and activation of n-type dopants in germanium, J. Appl. Phys. 104 (2008) 023523.

[5] C.O. Chui, K. Gopalakrishnan, P.B. Griffin, J.D. Plummer, K.C. Sarawat, Activation and diffusion studies of ion-implanted $\mathrm{p}$ and $\mathrm{n}$ dopants in germanium, Appl. Phys. Lett. 83 (2003) 3275

[6] A. Satta, T. Janssenss, T. Clarysse, E. Simoen, M. Meuris, A. Benedetti, I. Hoflijk, B. De Jaeger, C. Demeurisse, W. Vandervorst, P implantation doping of Ge: diffusion, activation, and recrystallization, J. Vac. Sci. Technol. 24 (2006) 494.

[7] A. Satta, E. Simoen, T. Janssenss, T. Clarysse, A. Benedetti, M. Meuris, W. Vandervorst, Diffusion, activation, and regrowth behavior of high dose $\mathrm{P}$ implants in Ge, Appl. Phys. Lett. 88 (2006) 162118.

[8] C.O. Chui, L. Kulig, J. Moran, W. Tsai, K.C. Saraswat, Germanium n-type shallow junction activation dependences, Appl. Phys. Lett. 87 (2005) 091909.

[9] A. Chroneos, D. Skarlatos, C. Tsamis, A. Christofi, D.S. McPhail, R. Hung, Implantation and diffusion of phosphorous in germanium, Mater. Sci. Semicond. Process. 9 (2006) 640.

[10] G.D. Dilliway, R. van den Boom, B. van Daele, F.E. Leys, T. Clarysse, B. Parmentier, A. Moussa, C. Defranoux, A. Benedetti, O. Richard, H. Bender, E. Simoen, M. Meuris, In-situ phosphorus doping of germanium by APCVD, ECS Trans. 3 (2006) 599. 
[11] J.M. Hartmann, J.-F. Damlencourt, Y. Bogumilowicz, P. Holliger, G. Rolland, T. Billon, Reduced pressure-chemical vapor deposition of intrinsic and doped Ge layers on $\mathrm{Si}(001)$ for microelectrics and optoelectronics purposes, J. Cryst. Growth 274 (2005) 90.

[12] H.-Y. Yu, S.-L. Cheng, P.B. Griffin, Y. Nishi, K.C. Saraswat, Germanium in situ doped epitaxial growth on $\mathrm{Si}$ for high-performance $\mathrm{n}^{+} / \mathrm{p}$-junction diode, IEEE Electron Device Lett. 30 (2009) 1002.

[13] A. Asaithambi, Using automatic differentiation to solve concentration dependent diffusion problems, Appl. Math. Comput. 159 (2004)
[14] M.S. Carroll, R. Koudelka, Accurate modelling of average phosphorus diffusivities in germanium after long thermal anneals: evidence of implant damage enhanced diffusivities, Semicond. Sci. Technol. 22 (2007) 164.

[15] P. Tsouroutas, D. Tsoukalas, I. Zergioti, N. Cherkashin, A. Claverie, Modeling and experiments on diffusion and activation of phosphorus in germanium, J. Appl. Phys. 105 (2009) 094910.

[16] A. Nayfeh, C.O. Chui, K.C. Saraswat, Effects of hydrogen annealing on heteroepitaxial-Ge layers on Si: surface roughness and electrical quality, Appl. Phys. Lett. 85 (2004) 2815. 